\title{
Use and misuse of the Kubelka-Munk function to obtain the band gap energy from diffuse reflectance measurements
}

\author{
Salmon Landi Jr. ${ }^{\text {a, }}$, Iran Rocha Segundo ${ }^{\text {b }}$, Elisabete Freitas ${ }^{\text {b }}$, Mikhail Vasilevskiy ${ }^{c, d}$, \\ Joaquim Carneiro $^{c}$, Carlos José Tavares ${ }^{c}$ \\ a Federal Institute Goiano, Rio Verde, Goiás, 75901-970, Brazil \\ b Civil Engineering Department, University of Minho, Guimarães, 4800-058, Portugal \\ ${ }^{\mathrm{c}}$ Centre of Physics of the Universities of Minho and Porto, Braga, 4710-057, Portugal \\ ${ }^{\mathrm{d}}$ International Iberian Nanotechnology Laboratory (INL), Av. Mestre José Veiga, 4715-330 Braga, Portugal
}

\section{A R T I C L E I N F O}

Communicated by: L. Brey

\section{Keywords:}

Diffuse reflectance

Kubelka-Munk model

Tauc plot

Band gap energy

\begin{abstract}
A B S T R A C T
The determination of the optical band gap energy $\left(E_{g}\right)$ is important for optimization of the generation of electron/hole pairs in semiconductor materials under illumination. For this purpose, the classical theory proposed by Kubelka and Munk (K-M) has been largely employed for the study of amorphous and polycrystalline materials. In this paper, the authors demonstrate, step by step, how to use the K-M function and apply it thoroughly to the determination of the $E_{g}$ of $\mathrm{TiO}_{2}$ semiconductor powder (pressed at different thicknesses) from diffuse reflectance spectroscopy (DRS) measurements. For the sample thicknesses $1-4 \mathrm{~mm}, E_{g}$ values of 3.12-3.14 eV were obtained. With this work it is envisaged a clarification to the procedure of determination of the $E_{g}$ from the K-M theory and DRS data, since some drawbacks, and misconceptions have been identified in the recent literature. In particular, the widely used practice of determining the $E_{g}$ of a material directly from the K-M function is found to be inadequate.
\end{abstract}

\section{Introduction}

Semiconductor materials have received increasing attention in the photocatalysis field because of their ability to degrade various pollutants present in water or air through a sequence of redox reactions [1-5]. The photocatalytic ability of semiconductors is related to the excitation of electrons from the valence band to the conduction band caused by the absorption of a photon with an appropriate wavelength. The photoexcited electrons and holes are good reductant and powerful oxidant species, respectively [6-8]. In this scenario, the application of semiconductor materials for environmental remediation in large scale will depend, among other factors, on its activation in an effective way by solar radiation. In this sense, the scientific community has investigated different strategies that allow a more efficient absorption of visible light by wide-gap semiconductors, such as $\mathrm{TiO}_{2}$ [9-12].

Diffuse Reflectance Spectroscopy (DRS) with a UV-visible spectrophotometer is a technique frequently employed to study the optical properties of solids. In this context, the classical Kubelka-Munk (K-M) model has been extensively employed to understand the light scattering from the surface of semiconductor powder materials [13,14]. Initially, Paul Kubelka and Franz Munk proposed a theoretical approach to study how the colour of a substrate is changed after the application of a paint layer with certain composition and thickness [15]. At present, the K-M model is commonly used to analyse DRS results and estimate the optical band gap energy (or simply the band gap) of semiconductor materials [16].

However, in a number of papers published recently, including some in prestigious journals in the field of Materials Science, some inconsistencies have been detected when the subject matter is the K-M model and the so-called Tauc plot [17-19]. Specifically, the most serious problem consists in determining of the band gap energy directly from the plot of the Kubelka-Munk function versus incident photon energy [20-23]. From the authors of this manuscript point of view, these oversimplified or even incorrect interpretations may confuse the readers concerning the applicability and validity of the K-M model, which are discussed in detail in this work.

\footnotetext{
* Corresponding author.

E-mail addresses: salmon.landi@ifgoiano.edu.br (S. Landi), iran_gomes@hotmail.com (I.R. Segundo), efreitas@civil.uminho.pt (E. Freitas), mikhail@fisica. uminho.pt (M. Vasilevskiy), carneiro@fisica.uminho.pt (J. Carneiro), ctavares@fisica.uminho.pt (C.J. Tavares).
} 
Therefore, the main objective of this work is to explain all the necessary steps to obtain the band gap from DRS data of a polycrystalline powder of the wide-gap semiconductor $\mathrm{TiO}_{2}$. Thus, with this work it is expected to help researchers who are starting their studies involving the determination of the band gap from DRS data and to clarify the procedure to those who could be confused due to the various drawbacks previously identified.

\section{Kubelka-Munk model}

Kubelka and Munk suggested that the absorption and scattering are first order phenomena [15]. The system in study consists of a substrate coated with a certain material of interest, which is illuminated with diffuse monochromatic radiation. Moreover, this system must have a disk form (cylindrical geometry) with a flat area $A$ and a thickness $L$, so boundaries' effect is neglected. The incident light has intensity $I$ and the reflected portion has intensity $J$, which allows to define a dimensionless quantity, the reflectance: $R=J / I$. Naturally, $R$ depends on $L$ and the absorption and scattering properties of the medium (Appendix).

When considering a sample with semi-infinite thickness $(L \rightarrow \infty)$, K-M model implies:

$\frac{K}{S}=\frac{(1-R)^{2}}{2 R}$

where $K$ and $S$ are the K-M absorption and scattering coefficients respectively. Eq. (1) provides the correct definition of the Kubelka-Munk function $(F(R)=K / S)$ [24]. It is important to emphasize that the Eq. (1) describes a particular case of the K-M model $(L \rightarrow \infty)$, which correspond physically to a medium that reflects the same amount of light regardless of the substrate reflectance value. Murphy affirms that typically a thickness of 1-3 mm is required [25]. In concordance, Escobedo-Morales et al. state that, in practice, thicknesses $>2 \mathrm{~mm}$ are sufficient to avoid any contribution from substrate [26].

Due to the fact that $K$ and $S$ have the units of inverse length, $F(R)$ is a dimensionless quantity. Therefore, $F(R)$ multiplied by the photon energy $(E)$, which appears frequently in the band gap determination from the DRS data, has, necessarily, the units of energy. Unfortunately, incorrect unit of $F(R) \times E$ has been identified [27] or the authors do not specify the respective unit [28-30], which is ambiguous.

Finally, the reader could question whether the model where the light travels in only one direction is appropriate to represent the diffuse reflection phenomenon. In this sense, a detailed study that accounts for the angular dependence of diffused scattering light intensity by a medium was published by Myrick et al. [31]. These authors showed that Eq. (1) applies also in three dimensions when $L \rightarrow \infty$, the scattering is isotropic and the medium is homogeneous.

\section{Absorption and scattering coefficients}

This section presents the dependence of the absorption $(\alpha)$ and scattering ( $s$ ) coefficients of a semiconductor (or insulator) material as a function of the incident photon energy ( $E$ ). In fact, $\alpha$ and $s$ are intrinsic optical properties of the materials and represent the probabilities of light being absorbed and scattered, respectively, per unit path length [32]. The standard assumption is that the absorption occurs essentially in the material while the scattering is due to material's inhomogeneity and, to the first approximation, may be considered as independent of the absorption. The theory of scattering by fluctuations of the dielectric constant in a non-absorbing material has been developed by several authors and, while these theoretical considerations may differ in details, the main result is the following [33]. If the characteristic scale of the inhomogeneities, $d$, is much smaller than the wavelength, $d \ll \lambda$, the scattering coefficient is proportional to the fourth power of the photon energy: $s \propto E^{4}$,

which is the case known as the Rayleigh scattering. In the opposite case, $\lambda \ll d$, a weaker dependence takes place:

$s \propto E^{2}$.

The spectral dependence of the absorption coefficients is specific for the type of investigated material. For semiconductors, the principal absorption mechanism is due to interband transitions and the absorption coefficient can be written in the following form [34]:

$\alpha(E) \propto \frac{\left(E-E_{g}\right)^{p}}{E}$,

where the exponent $p$ depends on the band structure of the semiconductor material and $E_{g}$ is an important parameter called the optical band gap energy and defined as the energy difference between the bottom of the conduction band and the top of the valence band. In particular, $p=1 / 2$ for dipole-allowed transitions occurring at a direct band gap, while $p=2$ for dipole-allowed transitions near an indirect gap where the participation of phonons is required. ${ }^{1}$ Besides that, there are also relevant cases of dipole-forbidden transitions (direct gap $p=3 / 2$ and indirect gap $p=3$ ), where the dipolar transitions are suppressed because the involved orbitals have the same parity. In general, such processes are much less likely (weak light absorption or emission) and are sometimes called weakly allowed transitions. As a very instructive example, we recommend reading the work published by Malerba et al. [35], which discusses the contributions of different transition types to the total absorption coefficient of bulk and thin film $\mathrm{Cu}_{2} \mathrm{O}$.

Using a statistical analysis of light propagation in media, Yang and Kruse proposed a revision to K-M theory by taking into account the effect of scattering on the path length of light propagation [36]. In this case, the authors demonstrated that the $K$ and $S$ parameters depend on the illumination geometry and, consequently, do not represent physical properties of materials. Anyway, Eqs. 35 and 36 in Ref. [36] allow us to conclude that $K$ and $S$ depend on the intrinsic absorption $\alpha$ and scattering $s$ coefficients of the material such that:

$\underset{s}{\alpha} \propto \frac{K}{S}$

In other words, it is not correct to interpret the K-M function (Eq. (1)) as the absorption coefficient [37]. Besides that, it is important to specify that the definition of $F(R)$ involves the K-M coefficients ( $K$ and $S$ ) in order to avoid a possible confusion with the $\alpha$ and $s$ coefficients [38-40].

Once the diffuse reflectance is measured, Eq. (1) yields the ratio between the K-M absorption and scattering coefficients. Combining Eqs. (1)-(4) and taking into account the power-law energy dependence of the scattering coefficient as $s \propto E^{q}$ (where $q=2$ or 4 , according to Eq. (2)), it is possible to write:

$F(R) \propto \frac{\left(E-E_{g}\right)^{p} / E}{E^{q}}$.

In the vicinity of $E_{g}$, it is possible to approximate the relatively slowly varying factor $E^{q}$, in comparison to the $\alpha$, by a constant. In other words, the scattering phenomenon has been neglected ( $s$ and $S$ are being considered as constants). This allows us to write

$(F(R) \times E)^{\frac{1}{p}}=A\left(E-E_{g}\right)$,

\footnotetext{
${ }^{1}$ We would like to highlight a recent work on the direct excitation (i.e., without phonon assistance) in silicon, an indirect band gap semiconductor, with an optical near-field. The authors even state that their results are important for progress in the development of alternative optical devices made of conventional silicon semiconductors [48].
} 
where $A$ is a proportionality constant independent of the photon energy.

For amorphous and polycrystalline materials, the density of states is non-zero inside the gap, with disorder-induced band tails, so the band gap width is not well defined. Band tail states are, in fact, electronic states present just above the valence band or right below the conduction band. In general, these states originate from thermal, structural, impurity and/or compositional disorder [41]. So, for these materials, the common procedure is to use the so-called Tauc plot obtained by extrapolating the dependence Eq. (6) to zero (while the real absorption coefficient for those energies is still non-zero because of the band tails) [42]. That is, it is clear from Eq. (6) that the $E_{g}$ must be obtained by extrapolating to zero a linear fit to a plot of $(F(R) \times E)^{1 / p}$ versus $E$. In general, the authors take the liberty of using the "Tauc plot" designation in other similar plots that also aim to obtain $E_{g}$ by extrapolation.

\section{Application of K-M function and Tauc plot to determine the band gap energy}

The main goal of this section is to emphasize that the determination of $E_{g}$ from the plot $F(R)$ versus $E$ is an inadequate procedure, although recently practiced by several authors [20-23]. The $E_{g}$ of commercial $\mathrm{TiO}_{2}$ nanoparticles will be calculated from diffuse reflectance data analysed according to Eqs. (1) and (6). For this purpose, diffuse reflectance spectroscopy (DRS) measurements were carried out using a Shimadzu 2501 PC spectrophotometer equipped with an integrating sphere. Barium Sulphate $\left(\mathrm{BaSO}_{4}\right)$ was used as reference for the reflectance spectra, which means that the value of $R$ in Eq. (1) is actually taken to be the $R_{\text {sample }} / R_{\mathrm{BaSO}_{4}}$ ratio. $\mathrm{TiO}_{2}$ nanoparticles, consisting of a mixture of anatase (80\%) and rutile (20\%) crystalline phases, were inserted into the circular cavity of the sample support and then compressed by a glass rod. To analyse the impact of thickness in obtaining $F(R)$, different samples were compressed with $0.5,1.0,1.7,2.0,2.5$ and $4.0 \mathrm{~mm}$ layer thickness.

Particularly, it is interesting to determine whether the thickness of the material deposited on the support satisfies the condition utilized to obtain $F(R)$, i.e., $L$ tends to infinity. Physically, this means that an increase in the thickness of the material does not cause a significant change in its reflectance or analogously in the $F(R)$. Technically, it was verified if there are differences between the results of reflectance for the maximum sample thickness allowed by the support (the height of support cavity is equal to $4.0 \mathrm{~mm}$ ) in comparison with thinner thicknesses $(0.5,1.0,1.7,2.0,2.5 \mathrm{~mm})$. Fig. 1a illustrates the reflectance results of the $\mathrm{TiO}_{2}$ samples as a function of the incident radiation wavelength (in the range $300-750 \mathrm{~nm}$ ) obtained from DRS.

Practically, for $\lambda>430 \mathrm{~nm}$ all radiation is reflected by $\mathrm{TiO}_{2}$ ( $R \approx 100 \%$ ), for which reason the powders appears white. On the other hand, for $\lambda<300 \mathrm{~nm}$ the incident photons have the energy sufficient for the electron/hole generation and, therefore, are almost completely absorbed by the material (the reflectance is very low). In fact, the slight differences observed in the spectra are more evident for the whole wavelength range shown in Fig. 1b. It is noted that the curves are very close to each other, except for the one corresponding to the sample of thickness equal to $0.5 \mathrm{~mm}$, suggesting that the sample support reflectance may have affected the spectrum of this sample. Furthermore, it is possible conclude that the wavelength correspondent to the band gap energy, or absorption edge, is most likely located between $\sim 420 \mathrm{~nm}$ or $2.95 \mathrm{eV}$ (beginning of absorption of radiation by sample, which $R$ starts to decrease) and $\sim 320 \mathrm{~nm}$ or $3.88 \mathrm{eV}$ (value from which almost all radiation is absorbed, i.e., $R \approx 0$ ). However, as discussed previously, in the vicinity of the absorption edges ( 320 and $420 \mathrm{~nm}$ ), there is an influence of the band tails, which cannot be assessed by DRS measurements. From the data illustrated in Fig. 1, $(F(R) \times E)^{1 / 2}$ (Fig. 2a) and $F(R)$ (Fig. 2b) were plotted as a function of the incident photon energy, considering the wavelength range between $\sim 320$ and $\sim 420 \mathrm{~nm}$.

It is noteworthy to point out that the curves shown in Fig. 2a (Fig. 2b) present a more pronounced linearity in the range approximately between $\sim 3.2$ and $\sim 3.8 \mathrm{eV}(\sim 3.3$ and $\sim 3.8 \mathrm{eV})$. The correct procedure consists in plotting the $(F(R) \times E)^{1 / 2}$ (and not $F(R)$ )versus $E$ fitting the linear portion of this curve by a straight line, which the intercept divided by the slope (in modulus) provides the numerical value for $E_{g}$ (Table 1). The uncertainties (calculated from the linear fit parameters) in Table 1 were determined through error propagation calculations using the quotient rule.

As it is well known, anatase-rutile binary systems perform better in photocatalytic processes in terms of efficiency, compared to the case when only one of these isolated components is used [43]. So, there is an extensive range of band gap values presented in the scientific literature for $\mathrm{TiO}_{2}$ resulting from the combination of rutile and anatase phases. Also, the optical properties, in general, show some variations depending on the experimental conditions used to synthesize the samples, chemical

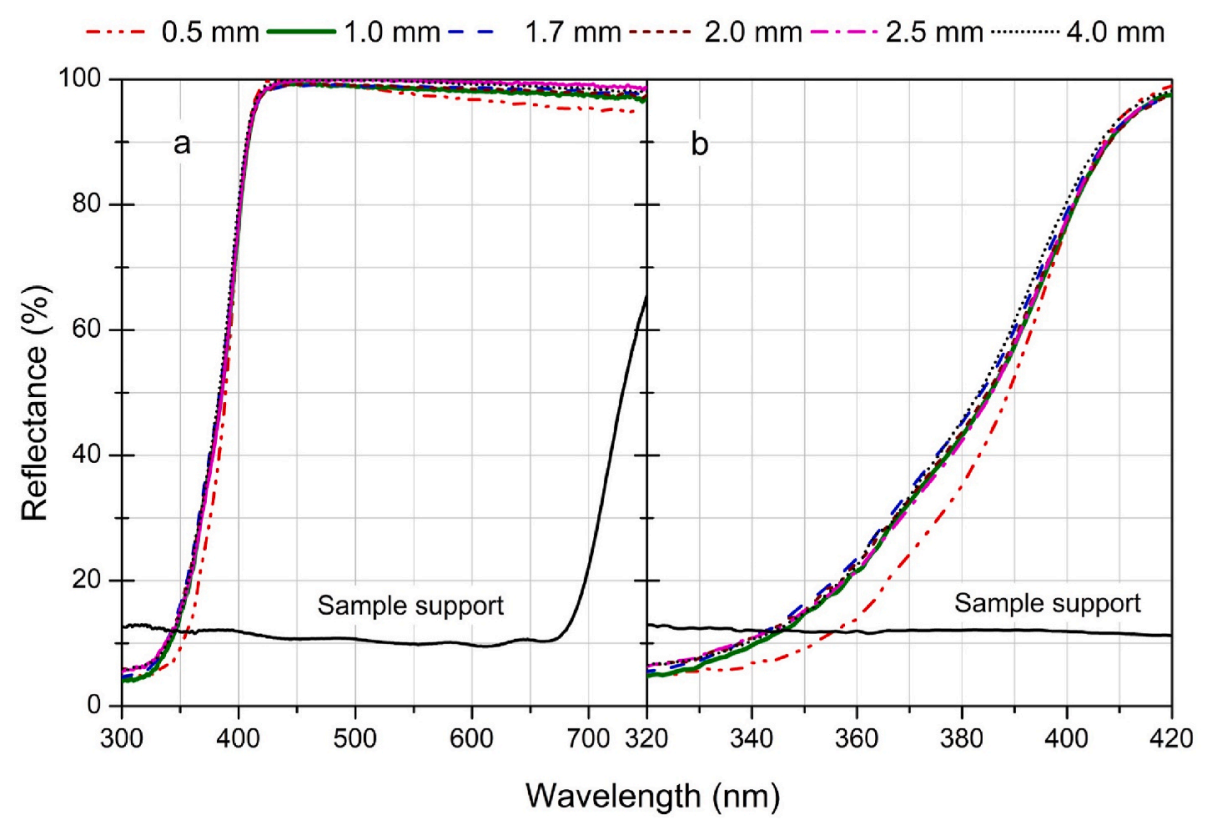

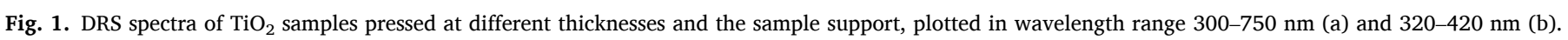




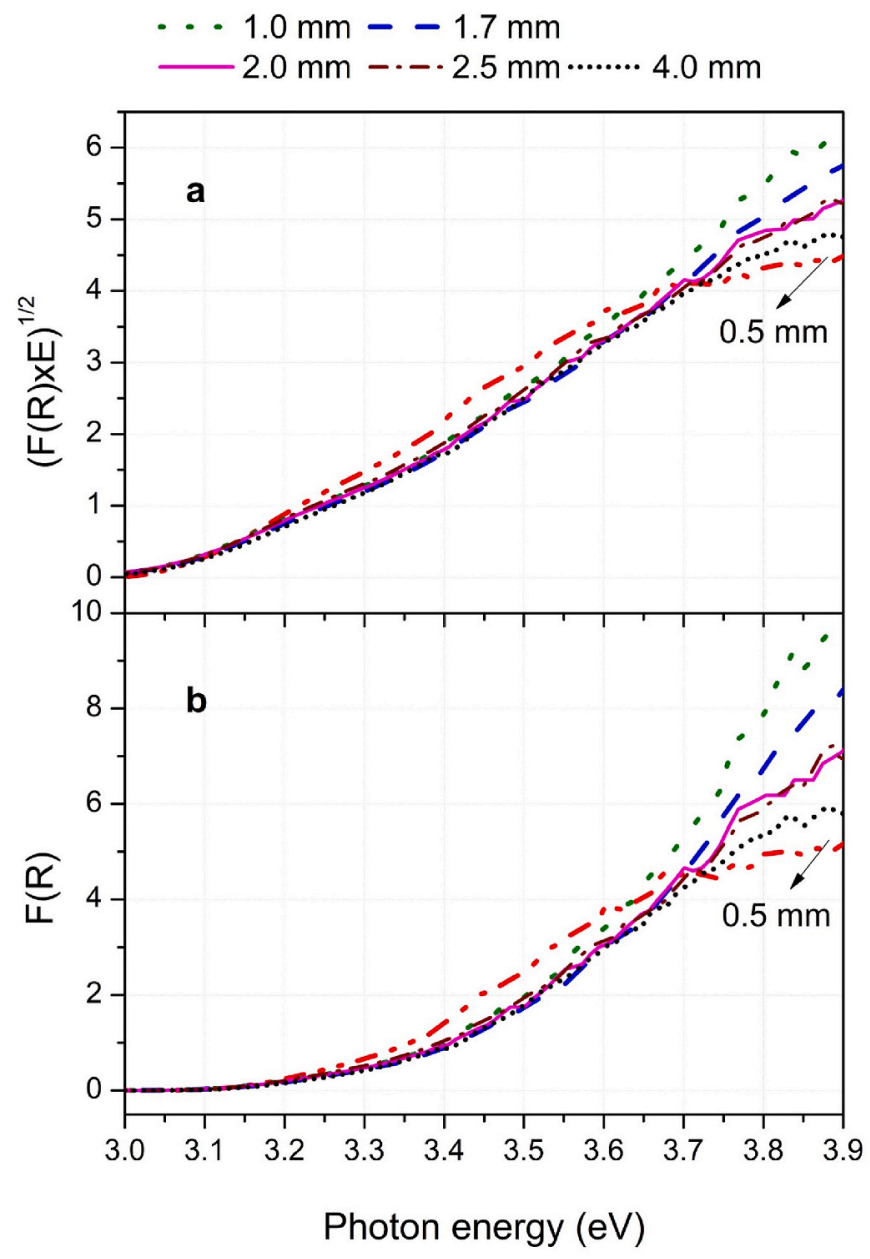

Fig. 2. Curves corresponding to the DRS patterns showed in Fig. 2 using: (a) Eq. (6) considering an indirect gap ( $p=2$ ) and (b) Eq. (1).

Table 1

Indirect band gap values for $\mathrm{TiO}_{2}$ powder layers with varying thicknesses calculated from the linear fits to $(F(R) \times E)^{1 / 2}$ versus $E$ (Fig. 2a, interval between 3.2 and $3.8 \mathrm{eV}$ ) and $F(R)$ versus $E$ (Fig. 2 b, interval between 3.3 and $3.8 \mathrm{eV}$ ).

\begin{tabular}{lll}
\hline Thickness $(\mathrm{mm})$ & $E_{g}(\mathrm{eV})$ from Fig. 2a & $E_{g}(\mathrm{eV})$ from Fig. 2b \\
\hline 0.5 & $3.05 \pm 0.03$ & $3.23 \pm 0.03$ \\
1.0 & $3.14 \pm 0.02$ & $3.33 \pm 0.05$ \\
1.7 & $3.14 \pm 0.02$ & $3.33 \pm 0.05$ \\
2.0 & $3.12 \pm 0.02$ & $3.32 \pm 0.04$ \\
2.5 & $3.11 \pm 0.01$ & $3.30 \pm 0.03$ \\
4.0 & $3.12 \pm 0.01$ & $3.31 \pm 0.03$ \\
\hline
\end{tabular}

composition and degree of disorder [44]. Howsoever, the band gap values obtained from the linear fits to $(F(R) \times E)^{1 / 2}$ versus $E$ are in reasonable agreement with those $(3.18 \mathrm{eV})$ reported by Rodríguez et al. [45], unlike the values presented in the last column of Table 1. Additionally, it is important to note that the $E_{g}$ obtained for $0.5 \mathrm{~mm}$ sample $(3.05 \mathrm{eV})$ is slightly lower than the expected value for non-doped $\mathrm{TiO}_{2}$ nanoparticles consisting of a mixture of anatase (80\%) and rutile (20\%) crystalline phases (3.19 eV) [46]. In fact, this thickness is not adequate to apply the K-M function (Eq. (1)), as explained in Section 2. This suggests that the $E_{g}$ reported by Polat is probably incorrect because the

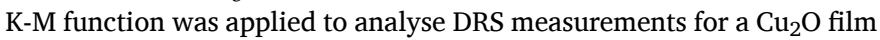
with a thickness of approximately $291 \mathrm{~nm}$ [47].

\section{Conclusion}

This work is aimed to discuss the K-M model and apply it to the analysis of diffuse reflectance data for a medium in form of powder pressed at different thicknesses. Also, the necessary steps to interpret $F(R)$ and its use for the determination of the band gap are presented and discussed in order to avoid any misunderstanding and inconsistencies. It is shown that, for a sample with a semi-infinite thickness, the $F(R)$ is defined as the ratio between the K-M absorption $(K)$ and scattering $(S)$ coefficients. Since these two coefficients have the same dimensions, the $\mathrm{K}-\mathrm{M}$ function is dimensionless.

Tauc plots were performed for pressed $\mathrm{TiO}_{2}$ nanoparticles with varying layer thickness in order to study the dependence of this property in the experimental determination of the band gap value. It was concluded that a thickness of about $0.5 \mathrm{~mm}$ is not sufficient for the use of the $F(R)$ in the investigated samples. Finally, the authors hope that this work can be useful to supress the various inconsistencies verified in recent works about the concepts involved in the application of the K-M model and Tauc plots.

\section{Declaration of competing interest}

The authors declare that they have no known competing financial interests or personal relationships that could have appeared to influence the work reported in this paper. 


\section{Appendix. Original derivation of the Kubelka-Munk function}

Kubelka and Munk proposed differential equations for the changes of incident light intensity going downward ( $i$ ) and going upward ( $j$ ) at any point $x$ within the film material medium [15]. At this moment, it is pertinent to answer the following question: how to calculate the reflectance for a layer of thickness $d x$ located at a distance $x$ from the substrate, inside the material? For this, it is necessary to quantify the incident and reflected flux within $d x$ (Fig A1).

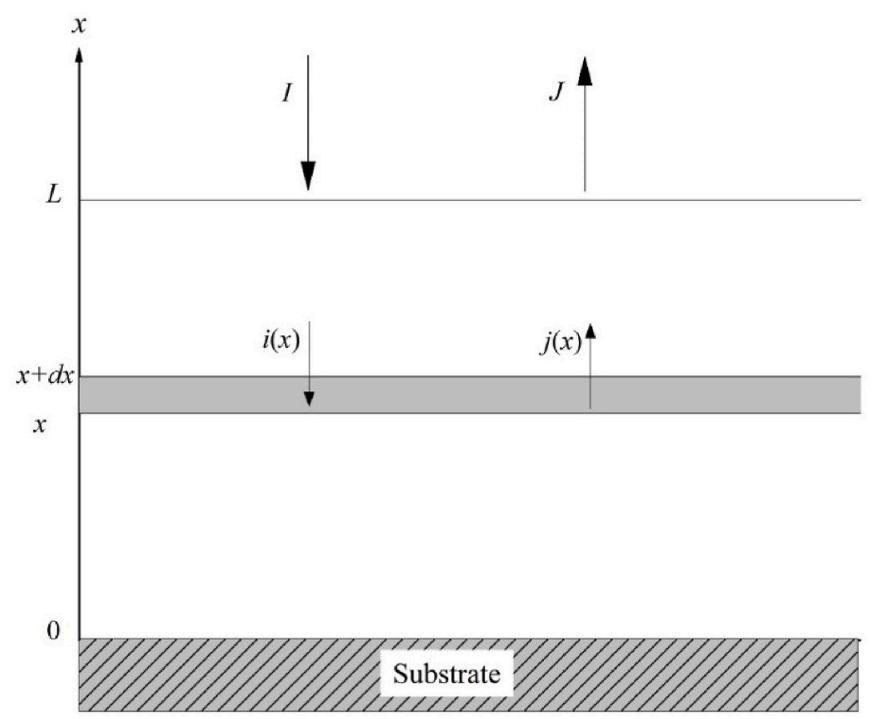

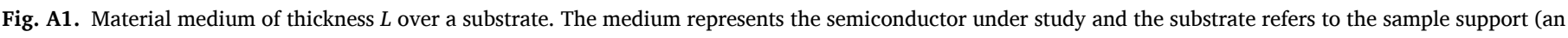
accessory utilized in DRS experiments of, consisting, in general, in a black metallic piece with a circular cavity to insert the sample).

The infinitesimal variation of $i(d i)$ is due to the: (I) material absorption presented within the volume of thickness $d x$ - in this case, $i$ suffers a decrease $(d i<0)$; (II) scattering of light going downward - $d i$ is also negative; and (III) scattering of light going upward - here, di is positive. After these considerations, the following equation is derived:

$d i=-\operatorname{Kidx}-\operatorname{Sid} x+\operatorname{Sjd} x$,

where $K$ and $S$ are the Kubelka-Munk absorption and scattering coefficients respectively; are positive and have units of inverse length. If the second and third members (in the right hand side of Eq. (A1)) are negligible, the Beer-Lambert equation is obtained. Similarly, the following equation for the change in light intensity going upward can be derived:

$d j=-K j d x-S j d x+S i d x$.

It is important to note that $d x$ has different signs in Eqs. A1 and A2. Therefore, when considering the positive $x$ axis oriented upwards, $d x$ is negative in Eq. (A1) and the di term on the left side of this equation must be replaced by - di. To solve these differential equations, it is useful to sum the results Eq. (A1) divided by $i$ (considering the correct signal) and Eq. (A2) divided by $j$, resulting in the Eq. (A3).

$\frac{d j}{j}-\frac{d i}{i}=-2(K+S) d x+S\left(\frac{j}{i}+\frac{i}{j}\right) d x$

Using the logarithm function properties and defining the reflectance of layer $d x$ as $r=j / i$ (analogous to $R=J / I$ ), Eq. (A3) can be written as:

$d(\ln r)=-2(K+S) d x+S\left(r+\frac{1}{r}\right) d x$

$\frac{d r}{r}=-2(K+S) d x+S\left(r+\frac{1}{r}\right) d x$

$d r=\left[r^{2}-2\left(\frac{K+S}{S}\right) r+1\right] S d x$

$\frac{d r}{\left[r^{2}-2\left(\frac{K+S}{S}\right) r+1\right]}=S d x$.

Defining $K / S+1=a$ (constant for an optically homogeneous medium [36]) and integrating over the entire thickness of the sample, Eq. (A4d) can be written as:

$\int_{R_{s}}^{R} \frac{d r}{r^{2}-2 a r+1}=S L$ 
where $R$ and $R_{S}$ are the reflectance on the coating surface (i.e. of the sample itself) and the substrate (sample support), respectively. The integral from Eq. (A5) can be solved by the partial fractions method, yielding the following result:

$\frac{1}{2 \sqrt{a^{2}-1}} \ln \left[\left(\frac{R-a-\sqrt{a^{2}-1}}{R-a+\sqrt{a^{2}-1}}\right)\left(\frac{R_{s}-a+\sqrt{a^{2}-1}}{R_{s}-a-\sqrt{a^{2}-1}}\right)\right]=S L$.

When considering a sample with semi-infinite thickness $(L \rightarrow \infty)$, practically speaking, a layer that is sufficiently thick to "hinder" the substrate influence, Eq. (A6) implies:

$R-a+\sqrt{a^{2}-1}=0$.

Rearranging Eq. (A7), Eq. (A8) becomes:

$\frac{K}{S}=\frac{(1-R)^{2}}{2 R} \equiv F(R)$

which is the K-M function, $F(R)$.

\section{Author contributions}

Salmon Landi Jr: Conceptualization, Writing - original draft preparation, Project administration. Iran Rocha Segundo: Methodology, Investigation, Data curation. Elisabete Freitas: Validation, Supervision. Mikhail Vasilevskiy: Writing - original draft preparation. Joaquim Carneiro: Resources. Carlos José Tavares: Writing - Reviewing \& Editing.

\section{References}

[1] H. Derikvandi, A. Nezamzadeh-Ejhieh, A comprehensive study on electrochemical and photocatalytic activity of $\mathrm{SnO}_{2}-\mathrm{ZnO} /$ clinoptilolite nanoparticles, J. Mol. Catal. Chem. 426 (2017) 158-169, https://doi.org/10.1016/j.molcata.2016.11.011.

[2] A. Rahmani-Aliabadi, A. Nezamzadeh-Ejhieh, A visible light $\mathrm{FeS} / \mathrm{Fe}_{2} \mathrm{~S}_{3}$ /zeolite photocatalyst towards photodegradation of ciprofloxacin, J. Photochem. Photobiol., A 357 (2018) 1-10, https://doi.org/10.1016/j. jphotochem.2018.02.006.

[3] H. Derikvandi, A. Nezamzadeh-Ejhieh, Comprehensive study on enhanced photocatalytic activity of heterojunction ZnS-NiS/zeolite nanoparticles: experimental design based on response surface methodology (RSM), impedance spectroscopy and GC-MASS studies, J. Colloid Interface Sci. 490 (2017) 652-664, https://doi.org/10.1016/j.jcis.2016.11.105.

[4] L. de Oliveira Pereira, I. Marques Sales, L. Pereira Zampiere, S. Silveira Vieira, I. do Rosário Guimarães, F. Magalhães, Preparation of magnetic photocatalysts from $\mathrm{TiO}_{2}$, activated carbon and iron nitrate for environmental remediation, J. Photochem. Photobiol., A 382 (2019) 111907, https://doi.org/10.1016/j. jphotochem.2019.111907.

[5] H. Yu, W. Dai, G. Qian, X. Gong, D. Zhou, X. Li, X. Zhou, The $\mathrm{NO}_{\mathrm{x}}$ degradation performance of nano- $\mathrm{TiO}_{2}$ coating for asphalt pavement, Nanomaterials 10 (2020) 897, https://doi.org/10.3390/nano10050897.

[6] A. Yousefi, A. Nezamzadeh-Ejhieh, M. Mirmohammadi, $\mathrm{SnO}_{2}-\mathrm{BiVO}_{4}$ mixed catalyst: characterization and kinetics study of the photodegradation of phenazopyridine, Environ. Technol. \& Innovation 22 (2021) 101433, https://doi. org/10.1016/j.eti.2021.101433.

[7] A. Noruozi, A. Nezamzadeh-Ejhieh, Preparation, characterization, and investigation of the catalytic property of $\alpha-\mathrm{Fe}_{2} \mathrm{O}_{3}-\mathrm{ZnO}$ nanoparticles in the photodegradation and mineralization of methylene blue, Chem. Phys. Lett. 752 (2020) 137587, https://doi.org/10.1016/j.cplett.2020.137587.

[8] N. Pourshirband, A. Nezamzadeh-Ejhieh, S.N. Mirsattari, The CdS/g- $\mathrm{C}_{3} \mathrm{~N}_{4}$ nanophotocatalyst: brief characterization and kinetic study of photodegradation and mineralization of methyl orange, Spectrochim. Acta Mol. Biomol. Spectrosc. 248 (2021) 119110, https://doi.org/10.1016/j.saa.2020.119110.

[9] M.A. Draz, H.H. El-Maghrabi, F.S. Soliman, H. Selim, A. Abdel Razik, A. El-sayed Amin, Y.M. Moustafa, A. Hamdy, A.A. Nada, Large scale hybrid magnetic $\mathrm{ZnFe}_{2} \mathrm{O}_{4} /$ $\mathrm{TiO}_{2}$ nanocomposite with highly photocatalytic activity for water splitting, J. Nanoparticle Res. 23 (1) (2021) 10, https://doi.org/10.1007/s11051-02005122-z.

[10] M. Olak-Kucharczyk, G. Szczepańska, M.H. Kudzin, M. Pisarek, The photocatalytical properties of $\mathrm{RGO} / \mathrm{TiO}_{2}$ coated fabrics, Coatings 10 (11) (2020) 1-15, https://doi.org/10.3390/coatings10111041.

[11] C.M. Magdalane, G.M.A. Priyadharsini, K. Kaviyarasu, A.I. Jothi, G. Gnanamani Simiyon, Synthesis and characterization of $\mathrm{TiO}_{2}$ doped cobalt ferrite nanoparticles via microwave method: investigation of photocatalytic performance of Congo red degradation dye, Surf. Interfaces 25 (2021) 101296, https://doi.org/10.1016/j. surfin.2021.101296.

[12] H. Zabihi-Mobarakeh, A. Nezamzadeh-Ejhieh, Application of supported $\mathrm{TiO}_{2}$ onto Iranian clinoptilolite nanoparticles in the photodegradation of mixture of aniline and 2, 4-dinitroaniline aqueous solution, J. Ind. Eng. Chem. 26 (2015) 315-321, https://doi.org/10.1016/j.jiec.2014.12.003Ind.Eng.Chem.

[13] N. Pourshirband, A. Nezamzadeh-Ejhieh, An efficient Z-scheme CdS/g- $\mathrm{C}_{3} \mathrm{~N}_{4}$ nano catalyst in methyl orange photodegradation: focus on the scavenging agent and mechanism, J. Mol. Liq. 335 (2021) 116543, https://doi.org/10.1016/j. molliq.2021.116543.

[14] N. Omrani, A. Nezamzadeh-Ejhieh, Focus on scavengers' effects and GC-MASS analysis of photodegradation intermediates of sulfasalazine by $\mathrm{Cu}_{2} \mathrm{O} / \mathrm{CdS}$ nanocomposite, Separ. Purif. Technol. 235 (2020) 116228, https://doi.org/ 10.1016/j.seppur.2019.116228.

[15] P. Kubelka, F. Munk, Ein beitrag zur optik der farbanstriche, Z. Techn. Phys. 12 (1931) 593-601.

[16] F. Soori, A. Nezamzadeh-Ejhieh, Synergistic effects of copper oxide-zeolite nanoparticles composite on photocatalytic degradation of 2,6-dimethylphenol aqueous solution, J. Mol. Liq. 255 (2018) 250-256, https://doi.org/10.1016/j. molliq.2018.01.169.

[17] S. Landi Jr., Comment on "Photocatalytic degradation of RhB from an aqueous solution using $\mathrm{Ag}_{3} \mathrm{PO}_{4} / \mathrm{N}-\mathrm{TiO}_{2}$ heterostructure" and "Evaluation of the effect of dose change of $\mathrm{Fe}_{3} \mathrm{O}_{4}$ nanoparticles on electrochemical biosensor compatibility using hydrogels as an experimental living organism model, J. Mol. Liq. 338 (2021) 116635, https://doi.org/10.1016/j.molliq.2021.116635.

[18] S. Landi Jr., Comment on "kubelka-munk function", Ceram. Int. 47 (2021) 8218-8227, https://doi.org/10.1016/j.ceramint.2021.06.103, and "Kubelka-Munk equation" - Ceram. Int. 47 (2021) 13980-13993, Ceram. Int. 47 (2021) 28055.

[19] S. Landi Jr., Comment on "molten salt synthesis of $\mathrm{Bi}_{2} \mathrm{WO}_{6}$ powders and its visiblelight photocatalytic activity" in materials research, 2019; 22(5): e20190311, Mater. Res. 24 (5) (2021), e20210193, https://doi.org/10.1590/1980-5373-MR2021-0193.

[20] K. Kucio, V. Sydorchuk, S. Khalameida, B. Charmas, The effect of mechanochemical, microwave and hydrothermal modification of precipitated $\mathrm{TiO}_{2}$ on its physical-chemical and photocatalytic properties, J. Alloys Compd. 862 (2021) 158011, https://doi.org/10.1016/j.jallcom.2020.158011.

[21] A. Meng, W. Tian, H. Yang, X. Wang, X. Wang, Z. Li, Molybdenum sulfide-modified metal-free graphitic carbon nitride/black phosphorus photocatalyst synthesized via high-energy ball-milling for efficient hydrogen evolution and hexavalent chromium reduction, J. Hazard Mater. 413 (2021) 125400, https://doi.org/ 10.1016/j.jhazmat.2021.125400.

[22] K. Kundu, P. Dutta, P. Acharyya, K. Biswas, Pb-free layered all-inorganic metal halides $\mathrm{RbSn}_{2} \mathrm{Br}_{5}$ : mechanochemical synthesis, band gap tuning, optical and dielectric properties, Mater. Res. Bull. 140 (2021) 111339, https://doi.org/ 10.1016/j.materresbull.2021.111339.

[23] N.O. Azarapin, V.V. Atuchin, N.G. Maximov, A.S. Aleksandrovsky, M.S. Molokeev, A.S. Oreshonkov, N.P. Shestakov, A.S. Krylov, T.M. Burkhanova, S. Mukherjee, O. V. Andreev, Synthesis, structure, melting and optical properties of three complex orthorhombic sulfides $\mathrm{BaDyCuS}_{3}, \mathrm{BaHoCuS}_{3}$ and $\mathrm{BaYbCuS}_{3}$, Matter. Res. Bull. 140 (2021) 111314, https://doi.org/10.1016/j.materresbull.2021.111314.

[24] M. Milosevic, S.L. Berets, A review of FT-IR of diffuse reflection sampling considerations, Appl. Spectrosc. Rev. 37 (4) (2002) 347-364, https://doi.org/ 10.1081/ASR-120016081.

[25] A.B. Murphy, Band-gap determination from diffuse reflectance measurements of semiconductor films, and application to photoelectrochemical water-splitting, Sol. Energy Mater. Sol. Cells 91 (14) (2007) 1326-1337, https://doi.org/10.1016/j. solmat.2007.05.005.

[26] A. Escobedo-Morales, I.I. Ruiz-López, M. de L. Ruiz-Peralta, L. Tepech-Carrillo, M. Sánchez-Cantú, J.E. Moreno-Orea, Automated method for the determination of the band gap energy of pure and mixed powder samples using diffuse reflectance spectroscopy, Heliyon 5 (2019), e01505, https://doi.org/10.1016/j.heliyon.2019. e01505. 
[27] A. Shawky, R.M. Mohamed, I.A. Mkhalid, M.A. Youssef, N.S. Awwad, Visible lightresponsive $\mathrm{Ag} / \mathrm{LaTiO}_{3}$ nanowire photocatalysts for efficient elimination of atrazine herbicide in water, J. Mol. Liq. 299 (2020) 112163, https://doi.org/10.1016/j. molliq.2019.112163.

[28] M. Momeni, H. Saghafian, F. Golestani-Fard, N. Barati, A. Khanahmadi, Effect of $\mathrm{SiO}_{2}$ addition on photocatalytic activity, water contact angle and mechanical stability of visible light activated $\mathrm{TiO}_{2}$ thin films applied on stainless steel by a sol gel method, Appl. Surf. Sci. 392 (2017) 80-87, https://doi.org/10.1016/j. apsusc.2016.08.165.

[29] D. Akcan, Effect of type and concentration of cobalt precursor on structural, optical and defect properties of $\mathrm{ZnCoO}$ nanoparticles, Mater. Res. Bull. 139 (2021) 111254, https://doi.org/10.1016/j.materresbull.2021.111254.

[30] J. Wawrzyniak, J. Karczewski, P. Kupracz, K. Grochowska, K. Załęski, O. Pshyk, et al., Laser-assisted modification of titanium dioxide nanotubes in a tilted mode as surface modification and patterning strategy, Appl. Surf. Sci. 508 (2020) 145143, https://doi.org/10.1016/j.apsusc.2019.145143.

[31] M.L. Myrick, M.N. Simcock, M. Baranowski, H. Brooke, S.L. Morgan, J. N. McCutcheon, The kubelka-munk diffuse reflectance formula revisited, Appl. Spectrosc. Rev. 46 (2) (2011) 140-165, https://doi.org/10.1080/ 05704928.2010.537004.

[32] L. Yang, B. Kruse, Revised Kubelka-Munk theory. I. Theory and application, J. Opt. Soc. Am. 21 (2004) 1933-1941, https://doi.org/10.1364/josaa.21.001933.

[33] H.G. Booker, W.E. Gordon, A theory of radio scattering in the troposphere Proceedings of the IRE 38 (4) (1950) 401-412, https://doi.org/10.1109/ JRPROC.1950.231435.

[34] P.K. Basu, Theory of Optical Processes in Semiconductors, Clarendon Press, Oxford, 1997.

[35] C. Malerba, F. Biccari, C.L.A. Ricardo, M. D’Incau, P. Scardi, A. Mittiga, Absorption coefficient of bulk and thin film $\mathrm{Cu}_{2} \mathrm{O}$, Sol. Energy Mater. Sol. Cells 95 (10) (2011) 2848-2854, https://doi.org/10.1016/j.solmat.2011.05.047.

[36] L. Yang, B. Kruse, S.J. Miklavcic, Revised Kubelka-Munk theory. II. Unified framework for homogeneous and inhomogeneous optical media, J. Opt. Soc. Am. A 21 (2004) 1942-1952, https://doi.org/10.1364/JOSAA.21.001942.

[37] K. Talukdar, B. Jun, Y. Yoon, Y. Kim, A. Fayyaz, C.M. Park, Novel Z-scheme $\mathrm{Ag}_{3} \mathrm{PO}_{4} / \mathrm{Fe}_{3} \mathrm{O}_{4}$-activated biochar photocatalyst with enhanced visible-light catalytic performance toward degradation of bisphenol A, J. Hazard Mater. 398 (2020) 123025, https://doi.org/10.1016/j.jhazmat.2020.123025.

[38] I. Martínez-Ruvalcaba, J.F. Hernández-Paz, J.R. Farías Mancilla, P. Piza Ruiz, C. A. Martínez Pérez, P.E. García-Casillas, C.A. Rodríguez-González, Optical properties of bio-inspired silver sulfide structures, J. Alloys Compd. 586 (2014) S526-S530, https://doi.org/10.1016/j.jallcom.2012.10.053.

[39] D.M. Tobaldi, R.C. Pullar, A.F. Gualtieri, G. Otero-Irurueta, M.K. Singh, M. P. Seabra, J.A. Labrincha, Nitrogen-modified nano-titania: true phase composition, microstructure and visible-light induced photocatalytic $\mathrm{NO}_{\mathrm{x}}$ abatement, J. Solid State Chem. 231 (2015) 87-100, https://doi.org/10.1016/j.jssc.2015.08.008.

[40] F. El Bachraouia, Y. Tamraouia, S. Louihib, J. Alamia, R. Shahbazian-Yassar, Y. Yuan, K. Amine, B. Manoun, Unusual superparamagnetic behavior in bulk $\mathrm{Ba}_{0.198} \mathrm{La}_{0.784} \mathrm{Ti}_{0.096} \mathrm{Fe}_{0.8} \mathrm{O}_{3-\delta}$, Mater. Res. Bull. 137 (2021) 111187, https://doi. org/10.1016/j.materresbull.2020.111187.

[41] J.F. Wager, Real- and reciprocal-space attributes of band tail states, AIP Adv. 7 (2017) 125321, https://doi.org/10.1063/1.5008521.

[42] J. Tauc, R. Grigorovici, A. Vancu, Optical properties and electronic structure of amorphous germanium, Phys. Status Solidi 15 (1966) 627-637, https://doi.org/ 10.1002/pssb.19660150224.

[43] Z. Sun, V.F. Pichugin, K.E. Evdokimov, M.E. Konishchev, M.S. Syrtanov, V. N. Kudiiarov, K. Li, S.I. Tverdokhlebov, Effect of nitrogen-doping and post annealing on wettability and band gap energy of $\mathrm{TiO}_{2}$ thin film, Appl. Surf. Sci. 500 (2020) 144048, https://doi.org/10.1016/j.apsusc.2019.144048.

[44] P. Kutálek, L. Tichý, On the thickness dependence of both the optical band gap and reversible photodarkening in amorphous Ge-Se films, Thin Solid Films 619 (2016) 336-341, https://doi.org/10.1016/j.tsf.2016.10.037.

[45] M.J.H. Rodríguez, E.P. Melián, O.G. Díaz, J. Araña, M. Macías, A.G. Orive, J.M. D. Rodríguez, Comparison of supported $\mathrm{TiO}_{2}$ catalysts in the photocatalytic degradation of $\mathrm{NO}_{\mathrm{x}}$, J. Mol. Catal. Chem. 413 (2016) 56-66, https://doi.org/ 10.1016/j.molcata.2015.12.007.

[46] S. Landi, J. Carneiro, O.S.G.P. Soares, M.F.R. Pereira, A.C. Gomes, A. Ribeiro, et al., Photocatalytic performance of $\mathrm{N}$-doped TiO2nano-SiO2-HY nanocomposites immobilized over cotton fabrics, J. Mater. Res. Technol. 8 (2019) 1933-1943, https://doi.org/10.1016/j.jmrt.2018.06.025.

[47] K. Polat, Cuprous oxide film sputtered on monolayer graphene for visible light sensitive heterogeneous photocatalysis, Thin Solid Films 709 (2020) 138254, https://doi.org/10.1016/j.tsf.2020.138254.

[48] M. Noda, K. Iida, M. Yamaguchi, T. Yatsui, K. Nobusada, Direct wave-vector excitation in an indirect-band-gap semiconductor of silicon with an optical nearfield, Phys. Rev. Appl. 11 (2019) 44053, https://doi.org/10.1103/ PhysRevApplied.11.044053. 